\title{
Nucleon observables and axial charges of other baryons using twisted mass fermions
}

\author{
Constantia Alexandrou* \\ Department of Physics, University of Cyprus, P.O. Box 20537, 1678 Nicosia, Cyprus, \\ Computation-based Science and Technology Research Center, Cyprus Institute, 20 Kavafi Str., \\ Nicosia 2121, Cyprus, \\ NIC, DESY, Platanenallee 6, D-15738 Zeuthen, Germany \\ E-mail: alexandeucy.ac.cy

\section{Martha Constantinou} \\ Department of Physics, University of Cyprus, P.O. Box 20537, 1678 Nicosia, Cyprus \\ E-mail: constantinou.martha@ucy.ac.cy

\section{Kyriakos Hadjiyiannakou} \\ Department of Physics, University of Cyprus, P.O. Box 20537, 1678 Nicosia, Cyprus \\ E-mail: hadjigiannakou.kyriakoseucy.ac.cy
}

\section{Karl Jansen}

NIC, DESY, Platanenallee 6, D-15738 Zeuthen, Germany

E-mail: karl.jansen@desy.de

\section{Christos Kallidonis}

Computation-based Science and Technology Research Center, Cyprus Institute, 20 Kavafi Str., Nicosia 2121, Cyprus

E-mail: c.kallidonis@cyi.ac.cy

\section{Giannis Koutsou}

Computation-based Science and Technology Research Center, Cyprus Institute, 20 Kavafi Str., Nicosia 2121, Cyprus

E-mail: g.koutsou@cyi.ac.cy

We present results on the nucleon scalar, axial and tensor charges, as well as, on the first moments of the unpolarized, polarized and transversity parton distributions using $N_{f}=2$ and $N_{f}=2+1+1$ twisted mass fermions. These include an ensemble that yields the physical value of the ratio of the nucleon to the pion mass. Results on the axial charges of hyperons and charmed baryons are also presented for a range of pion masses including the physical one.

32nd International Symposium on Lattice Field Theory - LATTICE 2014

23-28 June, 2014

Columbia University New York, NY

\footnotetext{
*Speaker.
} 


\section{Introduction}

We evaluate fundamental properties of the nucleon within the fermion twisted mass formulation of lattice QCD. The scalar, axial and tensor charges are computed using a number of twisted mass fermion (TMF) ensembles including one simulated with light quark masses fixed to their physical value. The computation of the nucleon axial charge allows a direct comparison with experiment, while determining the values of the scalar and tensor couplings provide useful input for searches beyond the familiar weak interactions of the Standard Model sought in the decay of ultracold neutrons. The computation of the tensor charge is particularly timely since new experiments using polarized ${ }^{3} \mathrm{He} /$ Proton at Jefferson lab aim at increasing the experimental accuracy by an order of magnitude [1]. In this work we use $N_{f}=2$ and $N_{f}=2+1+1$ ensembles of TMF with lattice spacings smaller than $0.1 \mathrm{fm}$ [2] to compute nucleon matrix elements at zero momentum transfer. The twisted mass formulation is well-suited for hadron structure calculations since it provides automatic $\mathscr{O}\left(a^{2}\right)$ improvement requiring no operator modification [3]. Our results include a simulation using the Iwasaki gluon action, and $N_{f}=2 \mathrm{TMF}$ with a clover term yielding approximately the physical value of the pion mass, on a lattice of size $48^{3} \times 96$, referred to as the physical ensemble [4].

Using similar techniques as those for the case of the nucleon, we also compute the axial charges of hyperons and charmed baryons on the same ensembles. The values of the axial charges of these particles are either poorly measured or not known and thus lattice QCD provides valuable input needed for phenomenological models.

\section{Hadron spectrum}
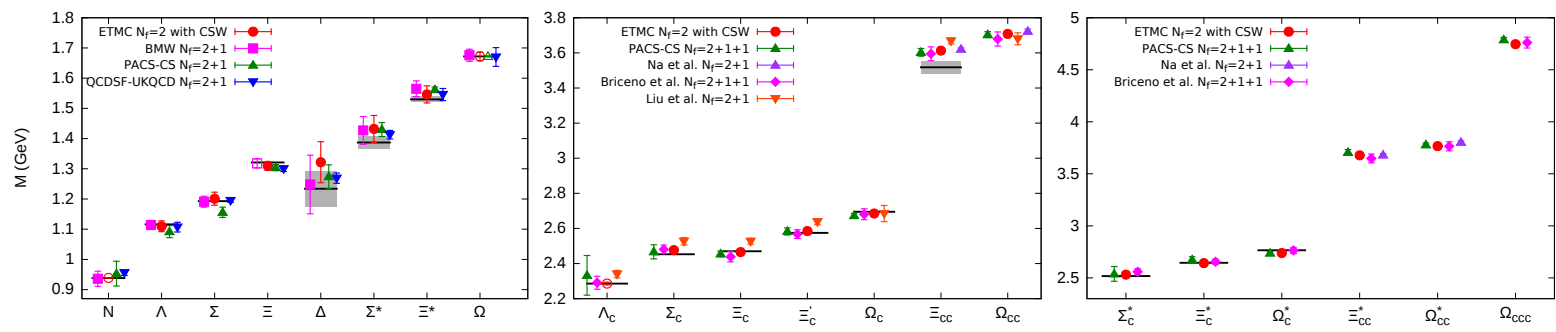

Figure 1: Results on baryon masses using the physical ensemble. Left: The octet and decuplet (the $\Omega$ and $\Xi$ were discovered at Brookhaven 50 years ago [7]). Spin-1/2 (middle) and spin-3/2 (right) charmed baryons.

Before discussing the structure of baryons we need to compute their mass. In Refs. [5, 6] the masses of baryons were investigated using $N_{f}=2$ and $N_{f}=2+1+1 \mathrm{TMF}$ ensembles for three lattice spacings and a range of pion masses the smallest one being $210 \mathrm{MeV}$. Here, we present results computed for the physical ensemble. The lattice spacing is $a=0.094(1) \mathrm{fm}$ determined from the nucleon mass. The strange and charm quark mass are fixed by using the $\Omega$ and $\Lambda_{c}$ mass, respectively. The resulting masses for the strange and charmed baryons are shown in Fig. 1. Although the continuum limit is not yet performed we observe agreement with the experimental values, as well as, with the values of other collaborations. Our previous study suggests that discretization errors are small and this may explain agreement with experiment even at a finite value of $a$. Assuming negligible cut-off effects, we can give a values for the yet non-measured mass of the charmed baryons. We find for the mass of $\Xi_{c c}^{*} 3.678(8) \mathrm{GeV}$, for the $\Omega_{c c}^{+} 3.708(10) \mathrm{GeV}$, for $\Omega_{c c}^{*+} 3.767(11) \mathrm{GeV}$ and for $\Omega_{c c c}^{++} 4.746(3) \mathrm{GeV}$. 


\section{Nucleon structure}

Results on the nucleon axial $g_{A}$, scalar $g_{S}$ and tensor $g_{T}$ charges and three first moments $\langle x\rangle_{q}$, $\langle x\rangle_{\Delta q}$ and $\langle x\rangle_{\delta q}$ are extracted by computing the forward nucleon matrix element $\left.\left\langle N\left(\vec{p}^{\prime}\right) \mathscr{O}_{X}^{a} N(\vec{p})\right\rangle\right|_{q^{2}=0}$. The operator $\mathscr{O}_{X}^{a}$ is $\bar{\psi}(x) \frac{\tau^{a}}{2} \psi(x)$ for the scalar, $\bar{\psi}(x) \gamma^{\mu} \gamma_{5} \frac{\tau^{a}}{2} \psi(x)$ for the axial-vector, and $\bar{\psi}(x) \sigma^{\mu \nu} \frac{\tau^{a}}{2} \psi(x)$ for the tensor charges, with the corresponding expressions for one derivative operators for the first moments. Here we present results for two ensembles: i) $N_{f}=2+1+1$ TMF on a $32^{3} \times 64$ lattice with $a=0.082 \mathrm{fm}$ and $m_{\pi}=373 \mathrm{MeV}$ (referred to as B55). A high statistics analysis is performed including disconnected contributions, using seven sink-source time separations ranging from $0.5 \mathrm{fm}$ to $1.5 \mathrm{fm}$, and ii) $N_{f}=2$ twisted mass with clover term on a $48^{3} \times 96$ lattice with $a=0.094(1) \mathrm{fm}$ and $m_{\pi}=130 \mathrm{MeV}$ (physical ensemble). A total of $\sim 1000$ confs are analyzed for three sink-source time separations ranging from about $0.9 \mathrm{fm}$ to $1.3 \mathrm{fm}$.
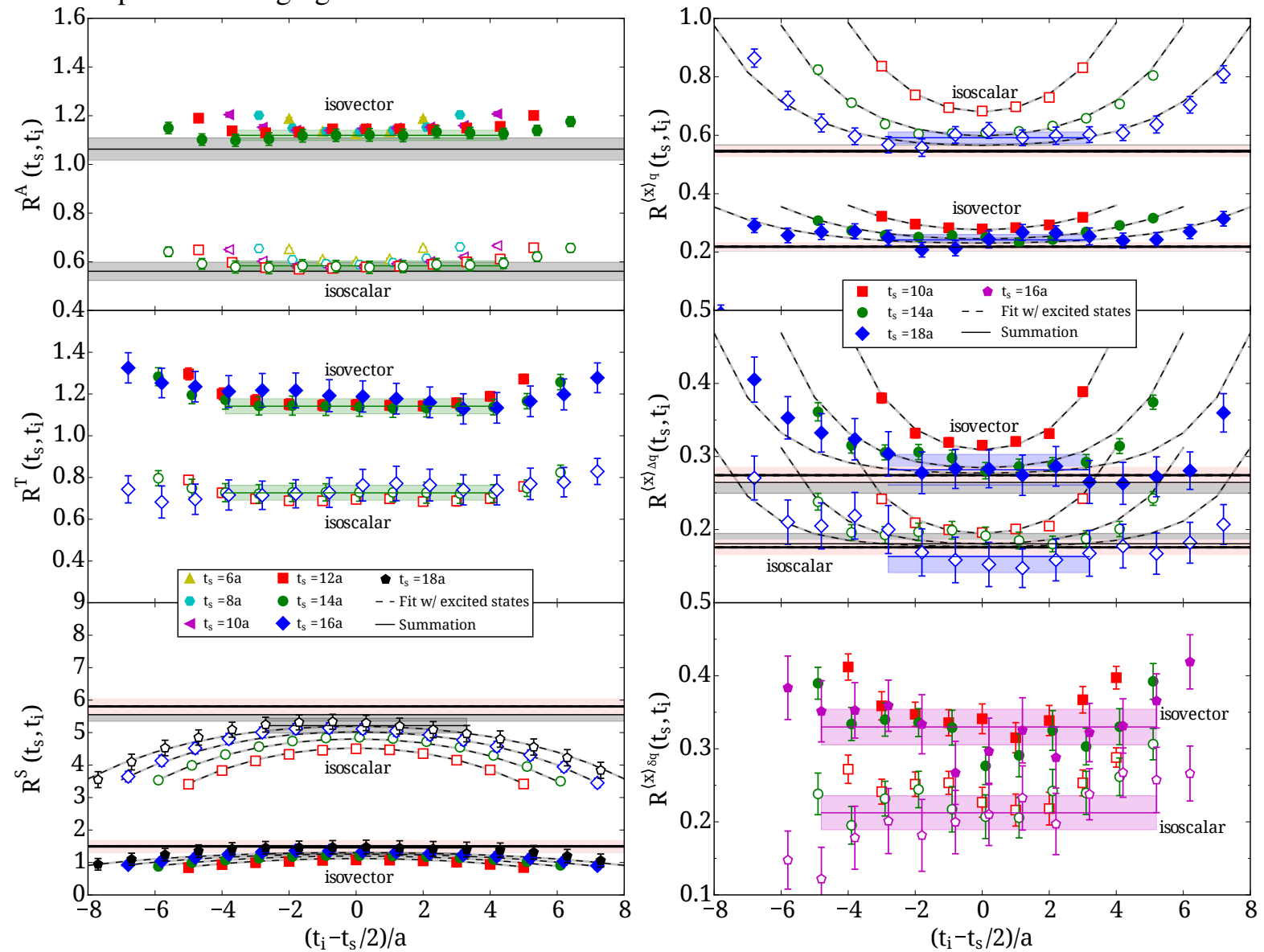

Figure 2: Results on the connected ratio $R\left(t_{s}, t_{\text {ins }}, t_{0}\right)$ for B55 using 1200 statistics for various $t_{s}$. Left panel: axial (top), tensor (middle) and scalar (bottom) currents. Right panel: one derivative vector, axial and tensor operators. The grey band is the result from the summation method. We also show simultaneous fits that take into account one excited state (dashed lines) yielding the value for the matrix element shown in light red.

The nucleon matrix element is extracted from the ratio $R\left(t_{s}, t_{\mathrm{ins}}, t_{0}\right)$ of the relevant three-point correlator divided by an appropriate combination of two-point functions [8] after identifying the nucleon ground state. Various approaches are applied to check ground state dominance: i) Plateau method:

$$
R\left(t_{s}, t_{\text {ins }}, t_{0}\right) \underset{\left(t_{\text {ins }}-t_{0}\right) \Delta \gg 1}{\left(t_{\text {ins }}\right) \Delta \gg 1} \mathscr{M}\left[1+\ldots e^{-\Delta(\mathbf{p})\left(t_{\text {ins }}-t_{0}\right)}+\ldots e^{-\Delta\left(\mathbf{p}^{\prime}\right)\left(t_{s}-t_{\text {ins }}\right)}\right]
$$



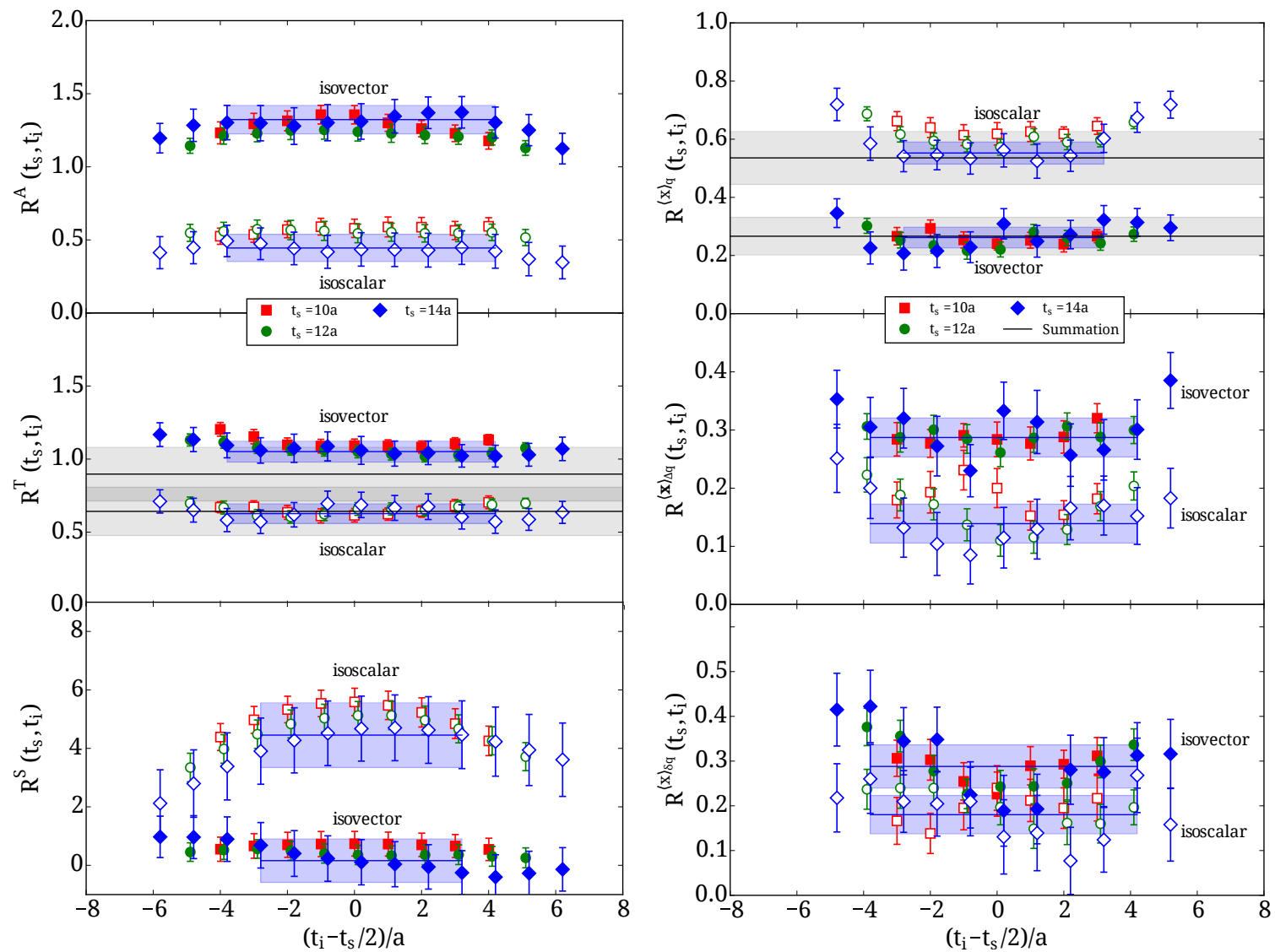

Figure 3: Results for the connected ratio $R\left(t_{s}, t_{\text {ins }}, t_{0}\right)$ for the physical ensemble for several $t_{s}$ using $\mathscr{O}\left(10^{3}\right)$ statistics. The notation is the same as in Fig. 2.

where $\mathscr{M}$ is the desired matrix element, $t_{s}, t_{\text {ins }}, t_{0}$ are the sink, insertion and source time-slices and $\Delta(\mathbf{p})$ the energy gap of the first excited state; ii) Summation method defined by summing over $t_{\text {ins }}$ : $\sum_{t_{\text {ins }}=t_{0}-1}^{t_{s}-1} R\left(t_{s}, t_{\text {ins }}, t_{0}\right)=$ Const. $+\mathscr{M}\left[\left(t_{s}-t_{0}\right)+\mathscr{O}\left(e^{-\Delta(\mathbf{p})\left(t_{s}-t_{0}\right)}\right)+\mathscr{O}\left(e^{-\Delta\left(\mathbf{p}^{\prime}\right)\left(t_{s}-t_{0}\right)}\right)\right]$. In this approach excited state contributions are suppressed by exponentials decaying with $t_{s}-t_{0}$, rather than $t_{s}-t_{\text {ins }}$ and $t_{\text {ins }}-t_{0}$. However, one needs to fit the slope rather than to a constant or take differences and then fit to a constant $[9,10]$. Both procedures generally lead to larger errors; iii) Perform fits including the contribution due to the first excited state.

Let us first examine the isovector combination, which has no disconnected contributions. In Fig. 2 we show the ratios from which $g_{A}, g_{T}$ and $g_{s}$ are extracted. As can be seen from analyzing a number of sink-source separations for the B55 ensemble, $g_{A}$ shows no detectable excited states and $g_{T}$ behaves similar to $g_{A}$ [11], while $g_{s}$ has severe contamination from excited states. The case of $g_{s}$ illustrates nicely that the plateau, summation and two-state fits all give consistent results. When this happens we have confidence in ground state dominance extracting a value that corresponds to the desired matrix element [12]. A similar analysis is performed for the first moments: $\langle x\rangle_{q}=$ $\int_{0}^{1} d x x[q(x)+\bar{q}(x)],\langle x\rangle_{\Delta q}=\int_{0}^{1} d x x[\Delta q(x)-\Delta \bar{q}(x)]$, and $\langle x\rangle_{\delta q}=\int_{0}^{1} d x x[\delta q(x)+\delta \bar{q}(x)]$, where $q(x)=q(x)_{\downarrow}+q(x)_{\uparrow}, \Delta q(x)=q(x)_{\downarrow}-q(x)_{\uparrow}, \delta q(x)=q(x)_{\perp}+q(x)_{\uparrow}$ for the one derivative vector, axial-vector and tensor operators respectively. Results on the three lowest moments obtained in the $\overline{\mathrm{MS}}$ at $4 \mathrm{GeV}^{2}$ are shown in Fig. 2 for B55. As can be seen, there is noticeable excited state 
contamination, especially for the isoscalar. Again consistency of the results extracted using the plateau and summation methods, and the two-state fit gives confidence in the final value. In Fig. 3 we show the corresponding analysis for the physical ensemble. Although the statistics is similar to that for B55 i.e. $\mathscr{O}\left(10^{3}\right)$ the errors are larger as expected, requiring more statistics to extract final numbers. Thus our results for the physical ensemble are to be regarded as preliminary.
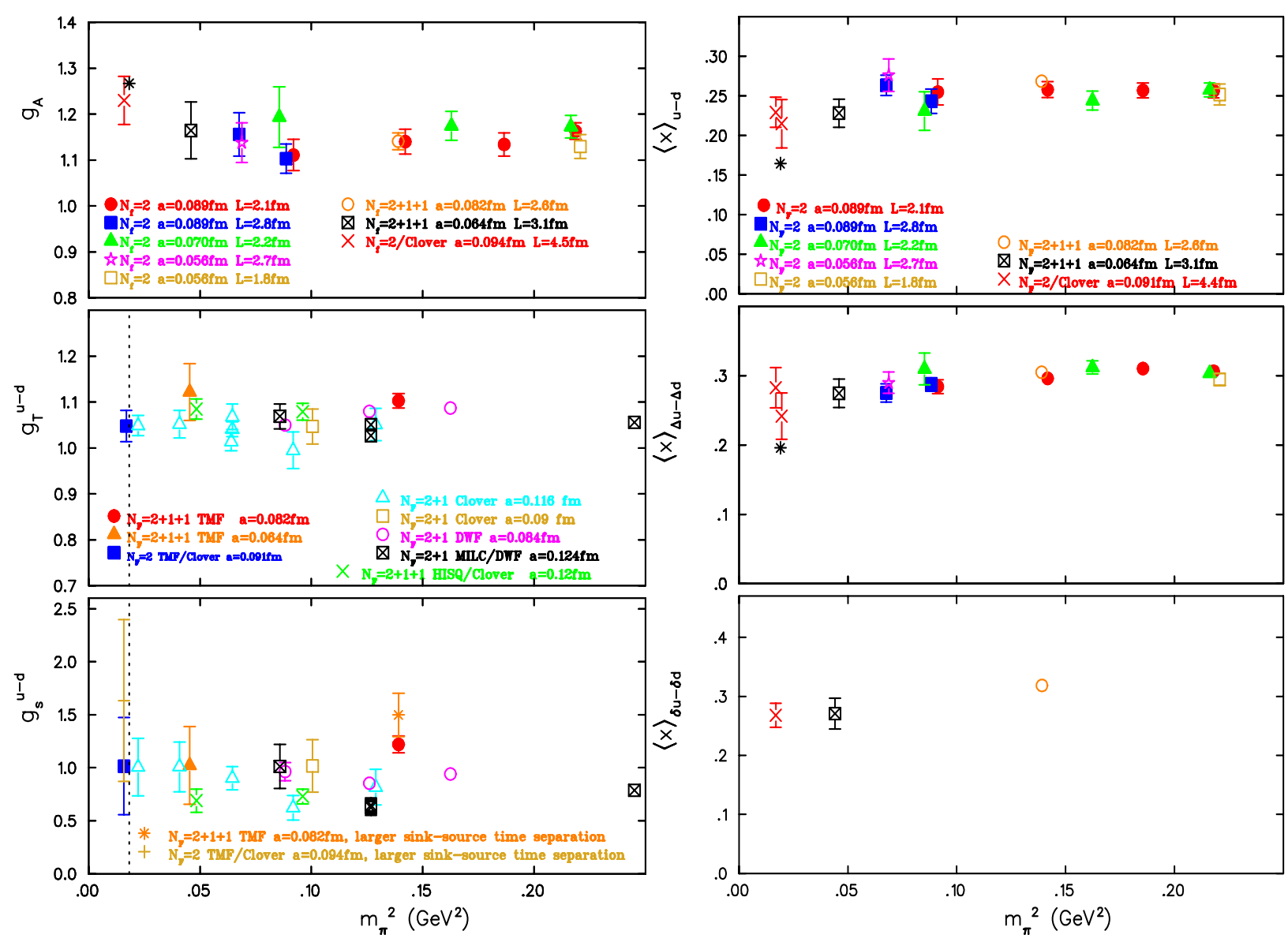

Figure 4: Nucleon isovector charges $g_{A}, g_{T}$ and $g_{s}$ (left) and first moments $\langle x\rangle_{q},\langle x\rangle_{\Delta q}$ and $\langle x\rangle_{\delta q}$ (right) versus $m_{\pi}^{2}$. TMF results at the physical point are preliminary. For $g_{T}$ and $g_{s}$ we include a comparison with the results of other collaborations. The orange asterisk and cross for $g_{s}$ at $m_{\pi}=373 \mathrm{MeV}$ and $130 \mathrm{MeV}$ respectively, is for $t_{s}-t_{0} \sim 1.5 \mathrm{fm}$, while the rest of the data are for $t_{s}-t_{0} \sim 1.1-1.2 \mathrm{fm}$.

In order to evaluate the isoscalar nucleon charges $g_{s}^{u+d}, g_{A}^{u+d}$ and $g_{T}^{u+d}$ one needs the disconnected contributions. For B55 these were computed for all local and one derivative operators using about 150,000 measurements on GPUs $[13,14,15,16,17]$. We found that disconnected contributions to $g_{A}^{u+d}$ are of the order of $10 \%$ while for $g_{T}^{u+d}$ are consistent with zero. The disconnected contributions to the $g_{s}^{u+d}$ arising from the light quark loops are larger than $10 \%$, while from the strange are smaller by a factor of about three. From this study, it is clear that we need to include the disconnected contributions in order to extract reliable results on $g_{A}^{u+d}$ and $g_{s}^{u+d}$.

In Fig. 4 we collect our results for the nucleon isovector charges and first moments for $t_{s}-t_{0} \sim$ $1.0-1.2 \mathrm{fm}$, which may be sufficient for quantities like $g_{A}$ but not for others such as $g_{s}$ where excited state contamination requires $t_{s}-t_{0}>1.5 \mathrm{fm}$. Indeed the agreement of $g_{A}$ at the physical 
pion mass with the experimental value indicates that such lattice artifacts are small. Given that $g_{T}$ shows similar behavior to $g_{A}$ the value found at the physical point can be taken as a prediction for the tensor charge of the nucleon. For $g_{s}$ one needs to increase the sink-source time separation to $\sim 1.5 \mathrm{fm}$ to ensure ground state dominance. Therefore, the results shown in Fig. 4 using $t_{s}-t_{0} \sim$ $1.1 \mathrm{fm}$ are only preliminary and we need to study $g_{s}$ as we increase $t_{s}-t_{0}$. Similarly $\langle x\rangle_{u-d}$ and $\langle x\rangle_{\Delta u-\Delta d}$ approach the physical value for bigger sink-source separations. In order to finalize their value one would need an equivalent high statistics study as the one performed at $m_{\pi}=373 \mathrm{MeV}$.

\section{Axial charges of hyperons and charmed baryons}
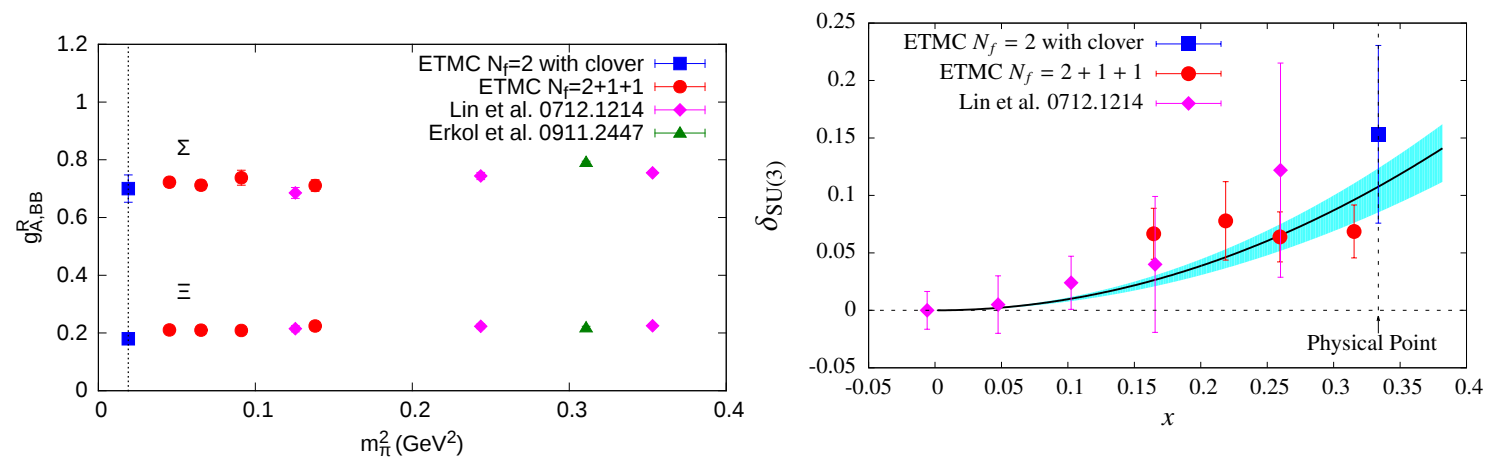

Figure 5: Left: Axial charge for the $\Sigma$ and $\Xi$ baryons versus $m_{\pi}^{2}$. Right: SU(3) breaking $\delta_{S U(3)}=g_{A}^{N}-g_{A}^{\Sigma}+$ $g_{A}^{\Xi}$ versus $x=\left(m_{K}^{2}-m_{\pi}^{2}\right) /\left(4 \pi^{2} f_{\pi}^{2}\right)$.
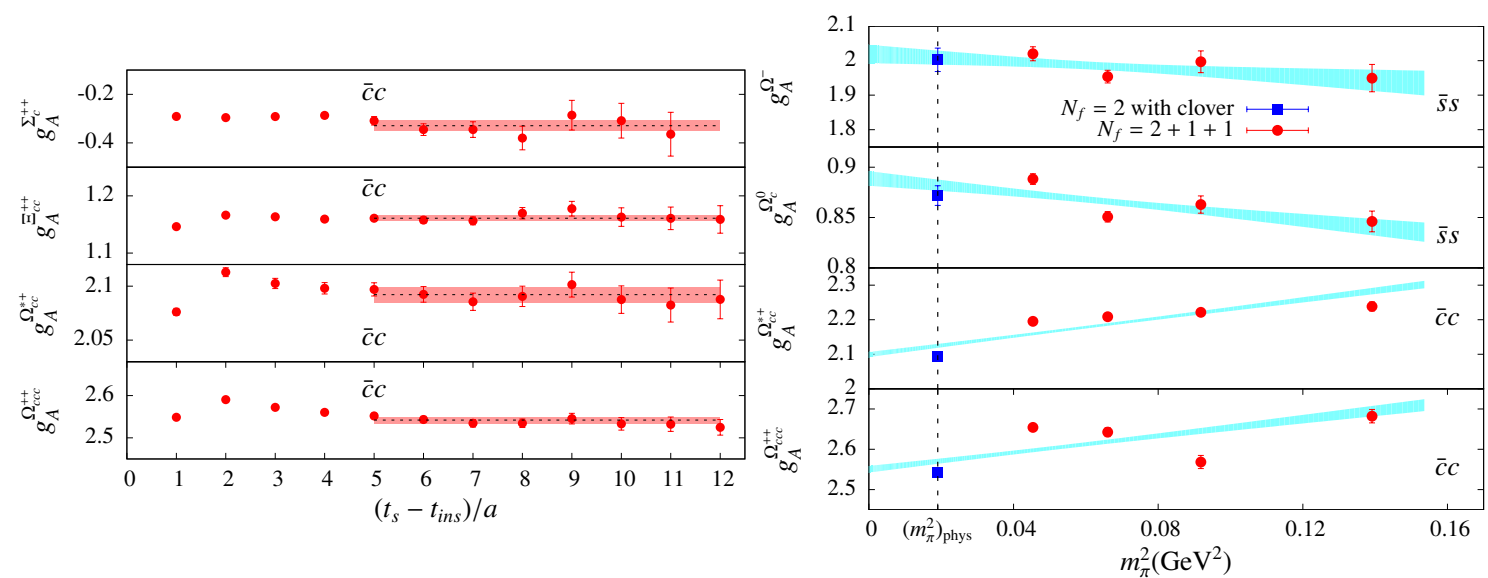

Figure 6: Left: Representative ratios from which the charmed contribution to the axial charge is extracted versus the sink time for fixed $t_{\text {ins. }}$. Right: Axial charges of the $\Omega$ baryons versus $m_{\pi}^{2}$.

Having the formalism to compute the nucleon axial charge one can use similar techniques to extract the axial charges of hyperons and charmed baryons. For this calculation we consider only connected contributions and use the fixed current method to evaluate the axial matrix elements $\left.\left\langle B\left(\vec{p}^{\prime}\right)\left|\bar{\psi}(x) \gamma_{\mu} \gamma_{5} \psi(x)\right| B(\vec{p})\right\rangle\right|_{q^{2}=0}$ performing only one sequential inversion per quark flavor for all baryons. In Fig. 5 we show representative results for the pion mass dependence of the axial charges of the $\Sigma$ and the $\Xi$ baryons. As can be seen, the dependence in $m_{\pi}^{2}$ is rather weak. In the same figure we also show the $\mathrm{SU}(3)$ breaking parameter $\delta_{\mathrm{SU}(3)} \equiv g_{A}^{N}-g_{A}^{\Sigma}+g_{A}^{\Xi}$. As one moves away from the $S U(3)$-symmetric point $\delta_{\mathrm{SU}(3)}$ increases reaching about 0.15 at the physical point. In Fig. 6 we 
show results on the axial charges of charmed baryons. The ratio $R\left(t_{s}, T_{\text {ins }}, t_{0}\right)$ at fixed $t_{\text {ins }} / a=7$ versus $t_{s}$ shows nice plateaus allowing a good determination of the axial charges. The axial charges of the $\Omega$ baryons show a linear dependence in $m_{\pi}^{2}$ yielding a result in complete agreement with the direct determination using our physical ensemble which is not included in the fit.

\section{Conclusions}

Simulations at the physical point are now feasible and this opens exciting possibilities for the study of hadron structure. In this work we show results on the nucleon charges, as well as, on the three first moments of PDFs. We find a value of $g_{A}$ that is in agreement with experiment. However, our results at the physical point highlight the need for larger statistics in order to carry out careful cross-checks. Noise reduction techniques such as all-mode-averaging, improved methods for disconnected diagrams and smearing techniques are currently being pursued aiming at decreasing our errors on the quantities obtained at the physical point. Predictions for other hadron observables are emerging as shown here for the case of the axial charges of hyperons and charmed baryons, as well as, on quantities probing beyond the standard model physics such as $g_{T}$ and $g_{s}$ from which the closely related $\sigma$-terms can be also extracted.

Acknowledgements: Partial support was provided by the projects EPYAN/0506/08, TECHNOL-

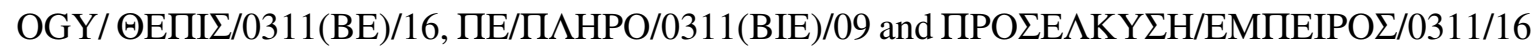
funded by the Cyprus Research Promotion Foundation.This work used computational resources provided by PRACE, JSC, Germany and the Cy-Tera project (NEA YПО $\Delta \mathrm{OMH} / \Sigma$ TPATH/0308/31).

\section{References}

[1] H. Gao et al., Eur. Phys. J. Plus 126, 2 (2011); Jefferson Lab experiment E12-11-108.

[2] P. .Boucaud et al. [ETM Collaboration], Phys. Lett. B 650, 304 (2007); R. Baron, et al. [ETM Collaboration] JHEP 1006, 111 (2010).

[3] R. Frezzotti and G. C. Rossi, JHEP 0408, 007 (2004) [hep-lat/0306014].

[4] A. Abdel-Rehim et al., PoS LATTICE 2013, 264 (2013) [arXiv:1311.4522].

[5] C. Alexandrou, V. Drach, K. Jansen, C. Kallidonis and G. Koutsou, arXiv:1406.4310 [hep-lat].

[6] C. Alexandrou, V. Drach, C. Kallidonis, G. Koutsou, PoS(Lattice2014).

[7] V. E. Barnes et al., Phys. Lett. 12, 134 (1964).

[8] C. Alexandrou et al. (ETMC) Phys. Rev. D 88, 014509 (2013) [arXiv:1303.5979].

[9] L. Maiani, G. Martinelli, M. L. Paciello and B. Taglienti, Nucl. Phys. B 293, 420 (1987).

[10] S. Capitani et al. (CLS), Phys. Rev. D 86, 074502 (2012) [arXiv:1205.0180].

[11] C. Alexandrou et al., PoS LATTICE 2013, 294, (2014).

[12] C. Alexandrou et al., PoS LATTICE 2013, 292 (2014) [arXiv:1312.2874].

[13] C. Alexandrou et al., Comput. Phys. Commun. 185, 1370 (2014) [arXiv:1309.2256].

[14] A. Abdel-Rehim et al., Phys. Rev. D 89, no. 3, 034501 (2014) [arXiv:1310.6339].

[15] C. Alexandrou et al. PoS LATTICE 2013, 411 (2014).

[16] C. Alexandrou, V. Drach, K. Jansen, G. Koutsou and A. Vaquero, PoS LATTICE 2013, 270 (2014).

[17] C. Alexandrou, et al., PoS LATTICE 2014, 140 (2015). 